TITLE:

\title{
Fabrication and transport properties for cleaved thin film BSCCO single crystals
}

\author{
AUTHOR(S):
}

Yamada, Y; Watanabe, T; Suzuki, M

\section{CITATION:}

Yamada, Y ... [et al]. Fabrication and transport properties for cleaved thin film BSCCO single crystals. IEEE TRANSACTIONS ON APPLIED SUPERCONDUCTIVITY 2007, 17(2): 35333536

\section{ISSUE DATE:}

2007-06

URL:

http://hdl.handle.net/2433/50220

\section{RIGHT:}

(c)2007 IEEE. Personal use of this material is permitted. However, permission to reprint/republish this material for advertising or promotional purposes or for creating new collective works for resale or redistribution to servers or lists, or to reuse any copyrighted component of this work in other works must be obtained from the IEEE. 


\title{
Fabrication and Transport Properties for Cleaved Thin Film BSCCO Single Crystals
}

\author{
Yoshiharu Yamada, Takao Watanabe, and Minoru Suzuki
}

\begin{abstract}
We have fabricated truly single-crystal BSCCO thin films of the quality of traveling-solvent-floating-zone single crystals by using a double side cleaving technique. The thicknesses of the cleaved thin films thus obtained ranges from $20 \mathrm{~nm}$ to $100 \mathrm{~nm}$. These films are found to adsorb or desorb oxygen very easily at a relatively low annealing temperature of 200 to $300^{\circ} \mathrm{C}$. When a cleaved film is annealed in Argon at $300^{\circ} \mathrm{C}$ for $10 \mathrm{~min}$, it changes to an insulator without any trace of superconducting transition. When this film is subsequently annealed in oxygen at $300^{\circ} \mathrm{C}$, it exhibits a sharp superconducting transition at about $90 \mathrm{~K}$. When the film is subjected to these process repeatedly, the film changes from an insulator to a superconductor quite reversibly. We have measured in-plane resistivity and the Hall coefficient systematically in a wide doping range for this thin film BSCCO single crystals.
\end{abstract}

Index Terms-Bi-based superconductor, doping dependence, excess oxygen, transport properties.

\section{INTRODUCTION}

$\mathbf{T}$ HE discovery of high $T_{c}$ superconductor (HTSC) has opened new prospects for applications in many areas including electronics. Without the necessity of liquid helium cooling the superconducting electronic devices have come to posses the potential for a widespread use. However, many difficulties have been pointed out in the fabrication of HTSC Josephson junctions, which are essential elements for electronics applications based on superconductors [1]. For example, the short coherence length of the HTSC materials requires extremely clean interfaces in Josephson junctions. At least as far as the Josephson devices are concerned, those with HTSC are not necessarily put to practical use so far. This is mainly because it is difficult to fabricate the Josephson junction made of HTSC whose performance is comparable to those with the conventional superconductor. On the other hand, it has been found that in strongly anisotropic HTSC such as $\mathrm{Bi}_{2} \mathrm{Sr}_{2} \mathrm{CaCu}_{2} \mathrm{O}_{8+\delta}(\mathrm{Bi} 2212)$, all $\mathrm{CuO}_{2}$ planes are connected by the Josephson effect [2]. These built-in tunnel junctions in a layered crystal structure are called the intrinsic Josephson junctions (IJJs). Among the various Josephson junctions with HTSC, the IJJ is found to be an ideal tunneling junction with a flat interface between superconductor and insulator on an atomic scale. An extensive study on IJJ is now under way to put them to practical use [3].

Manuscript received August 25, 2007. This work was supported in part by the 21st Century COE Program Grant from MEXT. The work of Y. Yamada was supported by the JSPS.

Y. Yamada and M. Suzuki are with the Department of Electronic Science and Engineering, Kyoto University, Nishikyo-ku, Kyoto 615-8510 Japan.

T. Watanabe is with the NTT Photonics Laboratories, Atsugi, Kanagawa 2430198 Japan.

Digital Object Identifier 10.1109/TASC.2007.899574
One major challenge in putting IJJs to practical use is to fabricate good quality thin films. It is rather difficult to deposit sufficiently good quality Bi-based superconducting thin films. Indeed, there are few publications which report the observation of resistive branches in the current-voltage curves for deposited thin films. On the other hand, the double side cleaving technique invented by Wang et al. a few years ago has allowed us to fabricate truly single-crystal BSCCO thin films from a bulk single crystal [4]. Such thin films are very important for not only applications but also basic research.

There has been much interest in studying the influence of oxygen content on the properties of HTSC, since superconductivity in cuprates is obtained by introducing carriers into the $\mathrm{CuO}_{2}$ planes of parent Mott insulators. For the Bi-based superconductor, control of the carrier concentration could be typically achieved by annealing samples in various atmospheres to alter the oxygen occupancy in the structure. However, it is rather difficult to control the carrier concentration in Bi-based single crystals in a wide range by annealing. Some of the annealing experiments for bulk single crystal Bi2212 have shown that $T_{c}$ could be depressed to $\sim 60 \mathrm{~K}$ in the underdoped region [5], [6], but only a few works have reported that $T_{c}$ could be reduced as low as $\sim 30 \mathrm{~K}$ [7]. In addition, it is hard to reduce $T_{c}$ below $30 \mathrm{~K}$ without introducing structural degradation of crystals. Moreover, annealed samples are sometimes found to be oxygen inhomogeneous, particularly for larger samples. These problems might be circumvented by using single-crystal BSCCO thin films fabricated by the double side cleaving technique.

By performing repeated annealing in an $\mathrm{Ar}$ or $\mathrm{O}_{2}$ flow on double side cleaved thin film sample, we have changed the carrier concentration systematically to an insulating region. Moreover, when the film is subjected to these process repeatedly, the film changes from an insulator to a superconductor quite reversibly. The reproducibility and the sensibility to the presence of oxygen of these cleaved films suggest that cleaved BSCCO films may be applicable to oxygen sensors. Furthermore, such samples would present an ideal stage for the systematic study of the Bi-based system by changing oxygen content. In this paper, we report a systematic measurement of the in-plane resistivity, $\rho_{a b}$, and the Hall coefficient, $R_{\mathrm{H}}$, at various doping levels from a nearly optimally doped region to an insulating region for cleaved thin film Bi2212 single crystals. It is found that the residual resistivity $\rho_{0}$ is relatively large near the superconductor-insulator (SI) transition region. In addition, the value for $R_{\mathrm{H}}$ at which superconductivity disappears is very small compared with the LSCO system [9] or the YBCO system [10], which implies that the reducing of the doping level in the Bi2212 system has additional influence on the occurrence of superconductivity more than the reducing of the carrier concentration. 

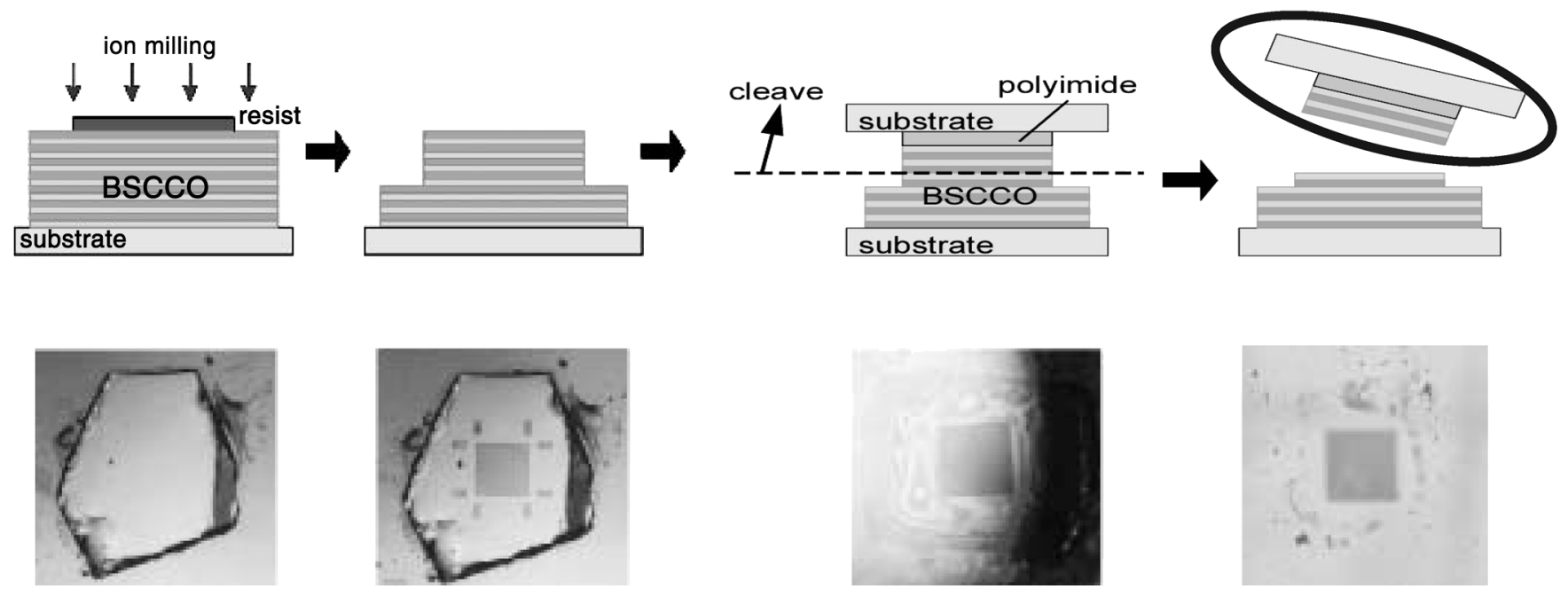

Fig. 1. Schematic description and optical image of the major steps in fabricating thin film Bi2212 single crystal by the double side cleaving process.

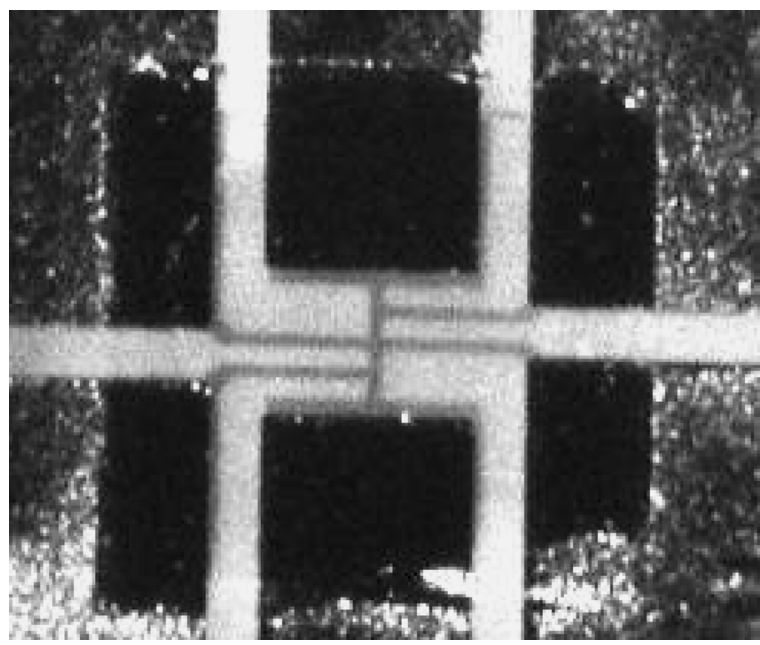

Fig. 2. Optical image of a sample after the film was patterned into a $10 \mu \mathrm{m}$ wide pattern with six terminals by chemical etching using dilute hydrochloric acid.

\section{SAMPLE FABRICATION}

Large single crystals of the Bi2212 compound were grown by the traveling-solvent floating-zone (TSFZ) method. The nominal composition of the starting material was $\mathrm{Bi}_{2.1} \mathrm{Sr}_{1.9} \mathrm{CaCu}_{2} \mathrm{O}_{8+\delta}$. Single crystal thin film samples were prepared in the following way. A cleaved TSFZ single crystal with a typical dimension of $1 \times 1 \times 0.03 \mathrm{~mm}^{3}$ was first glued on a sapphire substrate with stycast. A square mesa $300 \mu \mathrm{m}$ in side width was then prepared by standard photolithography and Ar ion milling. The surface of the patterned mesa was glued onto another sapphire substrate with polyimide, and then cleaved from the large pedestal. Fig. 1 shows the schematic description and optical image of the major steps in this process. The cleaved thin film glued on the sapphire substrate with a polyimide pedestal in between is a TSFZ single crystal itself. Then, the sample was patterned into a $10 \mu \mathrm{m}$ wide pattern with six terminals by chemical etching using dilute hydrochloric acid. Fig. 2 shows the optical image of a sample after the chemical etching. The thickness of the cleaved thin films thus

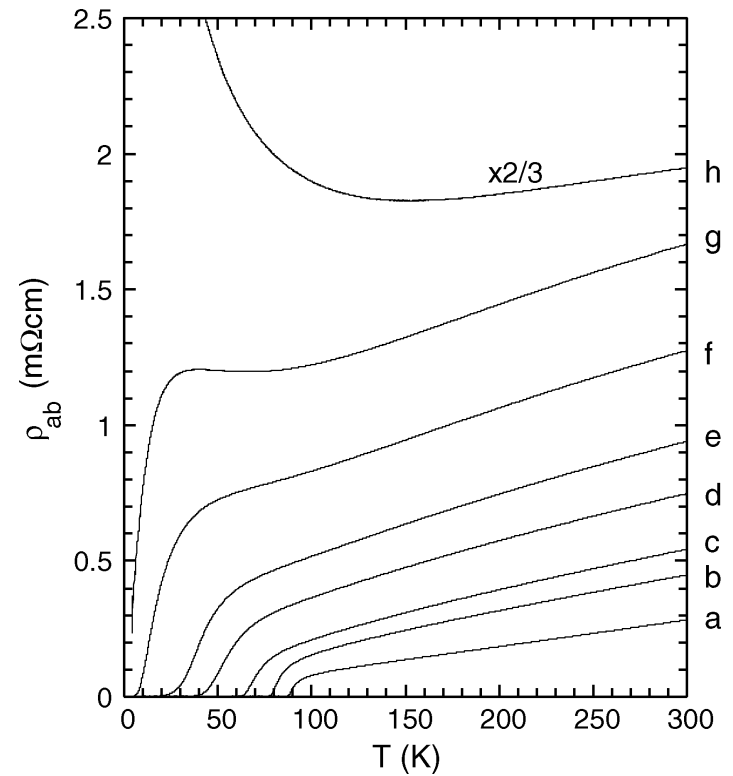

Fig. 3. The temperature dependence of in-plane resistivity for a Bi2212 single crystal thin film measured at various doping levels, which show systematic evolution with changing carrier concentration. The curve $a$ was obtained after the annealing for 10 minutes in flowing $\mathrm{O}_{2}$ at $300^{\circ} \mathrm{C}$ and other curves $(b-h)$ were obtained after the annealing for 10 minutes in flowing $\mathrm{Ar}$ at $220^{\circ} \mathrm{C}, 230^{\circ} \mathrm{C}$, $240^{\circ} \mathrm{C}, 250^{\circ} \mathrm{C}, 260^{\circ} \mathrm{C}, 270^{\circ} \mathrm{C}, 280^{\circ} \mathrm{C}$, respectively.

obtained ranged from $20 \mathrm{~nm}$ to $100 \mathrm{~nm}$. These films were found to adsorb or desorb oxygen very easily at a relatively low annealing temperature, $T$, of 200 to $300^{\circ} \mathrm{C}$. A standard ac six-probe method was employed to measure $\rho_{a b}$ and $R_{\mathrm{H}}$. Values for $R_{\mathrm{H}}$ were measured by sweeping the magnetic field for both plus and minus polarities at fixed temperatures.

\section{RESULTS AND DISCUSSION}

Fig. 3 shows the $T$ dependence of $\rho_{a b}$ for a Bi2212 cleaved single crystal obtained from a single sample by repeated annealing to change the carrier concentration, showing a systematic evolution of $\rho_{a b}$ from curve $a$ to $h$. The curve $a$ was obtained after the annealing for 10 minutes in flowing $\mathrm{O}_{2}$ at $300^{\circ} \mathrm{C}$. Other 


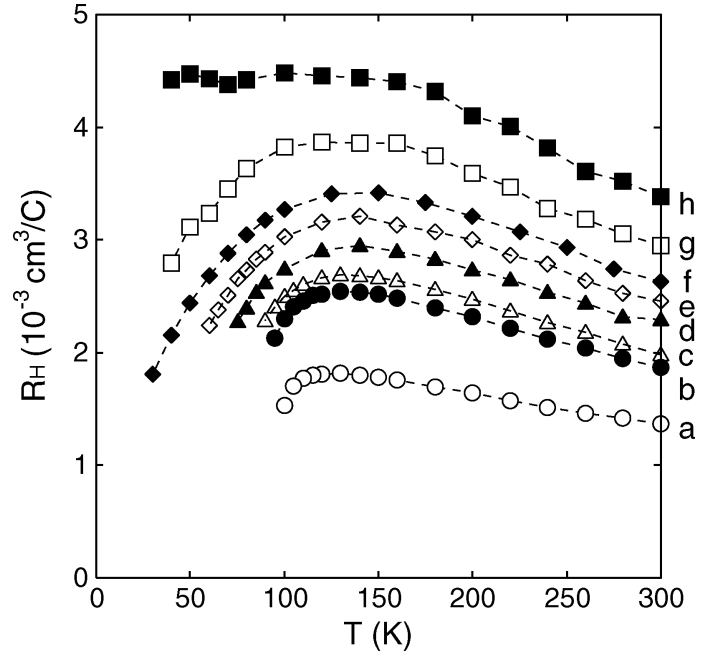

Fig. 4. Temperature dependence of $R_{\mathrm{H}}$ for the same sample as in Fig. 3. The systematic increase in $R_{\mathrm{H}}$ with decreasing oxygen is clearly seen, which suggests that the carrier concentration is actually reduced.

curves $(b-h)$ were obtained after the annealing for 10 minutes in flowing $\mathrm{Ar}$ at $220^{\circ} \mathrm{C}, 230^{\circ} \mathrm{C}, 240^{\circ} \mathrm{C}, 250^{\circ} \mathrm{C}, 260^{\circ} \mathrm{C}$, $270^{\circ} \mathrm{C}, 280^{\circ} \mathrm{C}$, respectively. Here we want to emphasize that we verified that the transport properties are quantitatively reproducible. In addition, the value for $\rho_{a b}$ of nearly optimum doping (curve $a$ ) is consistent with that of a bulk single crystal [5] in contrast to the case for films deposited by sputtering [11]. It is clearly seen that the magnitude of $\rho_{a b}$ and its slope $\left(d \rho_{a b} / d T\right)$ increase systematically with decreasing doping. In addition, the estimated $\rho_{0}$ becomes larger with decreasing doping although $\rho_{0}$ is approximately 0 at the nearly optimum doping level (curve a). Notably, the value for $\rho_{0}$ of curve $g$ in Fig. 3, which is near the SI transition, is $\sim 1 \mathrm{~m} \Omega \mathrm{cm}$. This value corresponds to the two dimensional sheet resistance $\rho_{0}^{2 \mathrm{D}} \sim 6.7 \mathrm{k} \Omega$ per $\mathrm{CuO}_{2}$ bilayer, and is close to the universal value $h / 4 \mathrm{e}^{2} \simeq 6.5 \mathrm{k} \Omega$ [12]. This coincidence is also seen in the carrier concentration driven SI transition in the YBCO system [13].

Fig. 4 shows the $T$ dependence of $R_{\mathrm{H}}$ for the same sample as in Fig. 3. A systematic increase in $R_{\mathrm{H}}$ with decreasing oxygen is clearly seen, which suggests that the carrier concentration is actually reduced. In addition, $R_{\mathrm{H}}-T$ curves in the superconducting region $(a-g)$ have broad peaks, the positions of which are shifted to the higher $T$ with decreasing doping. This behavior may be relevant to the opening of the pseudogap [14]. On the other hand, in the insulating region $(h)$, it shows a $T$-independent behavior at low temperatures. Similar behaviors of $R_{\mathrm{H}}(T)$ are also observed in the bulk single crystals LSCO or YBCO system [13], [15].

Fig. 5 shows the plots for $\cot \theta_{\mathrm{H}}$ vs $T^{2}$ at an applied field of $1 \mathrm{~T}$. The data of $\cot \theta_{\mathrm{H}}$ in the superconducting region $(a-g)$ are almost linear in $T^{2}$. This is also similar to the case for single crystals of LSCO or YBCO system. On the other hand, it is clearly seen that the magnitude of $\cot \theta_{\mathrm{H}}$ appears to be parallel-shifted notably with decreasing doping even in the superconducting region. This is apparently at variance with the case for LSCO or YBCO system, in which the magnitude of $\cot \theta_{\mathrm{H}}$ in the superconducting region is almost independent of

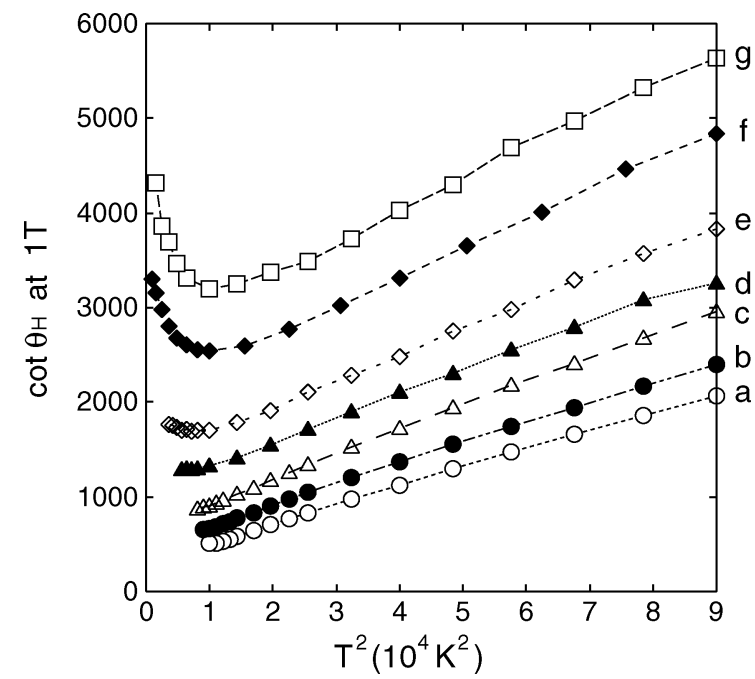

Fig. 5. Plots of $\cot \theta_{\mathrm{H}}$ vs $T^{2}$ for the data in superconducting region. It is clearly seen that the magnitude of $\cot \theta_{\mathrm{H}}$ is parallel-shifted notably with decreasing doping.

carrier concentration [9], [10]. This is because the magnitude of $R_{\mathrm{H}}$ at which superconductivity disappears is small compared with LSCO or YBCO system, while the magnitude of $\rho_{a b}$ is roughly the same. Semiquantitatively, $\rho_{a b}$ at $300 \mathrm{~K}$ changes from $\sim 0.3 \mathrm{~m} \Omega \mathrm{cm}$ at the optimum doping to $\sim 2 \mathrm{~m} \Omega \mathrm{cm}$ near the SI transition [16]. These values are common to the Bi2212, LSCO and YBCO systems. On the other hand, $R_{\mathrm{H}}$ at $300 \mathrm{~K}$ for Bi2212 changes from $\sim 1.4 \times 10^{-3} \mathrm{~cm}^{3} / \mathrm{C}$ at the optimum doping to $\sim 3.0 \times 10^{-3} \mathrm{~cm}^{3} / \mathrm{C}$ near the SI transition by a factor of $\sim 2$ while that for the LSCO or YBCO system changes from $\sim 1.0 \times 10^{-3} \mathrm{~cm}^{3} / \mathrm{C}$ at the optimum doping to $\sim 1.0 \times$ $10^{-2} \mathrm{~cm}^{3} / \mathrm{C}$ near the SI transition (by a factor of $\sim 10$ ) [9], [10]. This implies that $T_{c}$ for the oxygen reduced underdoped Bi2212 is anomalously suppressed.

It was reported that the inverse parabolic dependence of the $T_{c}$ on the oxygen content of Bi2212 is rather narrow in deposited thin films. In the present study, we have clarified that this is the case for TSFZ single crystals. Although the situation needs further clarification in detail, this anomalous suppression of $T_{c}$ in the underdoped region may be related to the disorder induced by excess oxygen atoms. Indeed recent numerical [17] and experimental [18] studies on excess oxygens suggest that these oxygens have an impact on the superconducting states.

\section{CONCLUSION}

We have fabricated truly single-crystal BSCCO thin films of the quality of traveling-solvent-floating-zone single crystals by a using double side cleaving technique. These films are found to adsorb or desorb oxygen very easily at a relatively low annealing temperature of 200 to 300 . When a cleaved film is annealed in $\mathrm{Ar}$ or $\mathrm{O}_{2}$ repeatedly, the film changes from an insulator to a superconductor quite reversibly. We have measured $\rho_{a b}$ and $R_{\mathrm{H}}$ systematically from a nearly optimally doped region to an insulating region for such thin film Bi2212 single crystal. It is found that the residual resistivity $\rho_{0}$ is relatively large near the SI transition region compared with the cation substituted systems. The value for $R_{\mathrm{H}}$ at which superconductivity disappears is 
very small compared with those of the LSCO or YBCO system, which implies that excess oxygen atoms have strong influence to suppress superconductivity in the underdoped region.

\section{REFERENCES}

[1] A. Barone and G. Paternò, Physics and Applications of the Josephson Effect. New York: John Wiley \& Sons, Inc., 1982.

[2] R. Kleiner and P. Müller, "Intrinsic Josephson effects in high- $T_{\mathrm{c}}$ superconductors," Phys. Rev. B, vol. 49, pp. 1327-1341, Jan. 1994.

[3] A. A. Yurgens, "Intrinsic Josephson junctions: Recent developments," Supercond. Sci. Technol., vol. 13, pp. R85-R100, Aug. 2000.

[4] H. B. Wang, P. H. Wu, and T. Yamashita, "Stacks of intrinsic Josephson junctions singled out from inside $\mathrm{Bi}_{2} \mathrm{Sr}_{2} \mathrm{CaCu}_{2} \mathrm{O}_{8+\delta}$ single crystals," Appl. Phys. Lett., vol. 78, pp. 4010-4012, Jun. 2001.

[5] T. Watanabe, T. Fujii, and A. Matsuda, "Anisotropic resistivities of precisely oxygen controlled single-crystal $\mathrm{Bi}_{2} \mathrm{Sr}_{2} \mathrm{CaCu}_{2} \mathrm{O}_{8+\delta}$ : Systematic study on "Spin Gap" effect," Phys. Rev. Lett., vol. 79, pp. 2113-2116, Sep. 1997.

[6] C. Kendziora, R. J. Kelly, E. Skelton, and M. Onellion, "Advances in single-crystal $\mathrm{Bi}_{2} \mathrm{Sr}_{2} \mathrm{CaCu}_{2} \mathrm{O}_{8+\delta}$ superconductors," Physica C, vol. 257, pp. 74-78, Jan. 1996.

[7] B. Liang, C. T. Lin, A. Maljuk, and Y. Yan, "Effect of vacuum annealing on the structure and superconductivity of $\mathrm{Bi}_{2} \mathrm{Sr}_{2} \mathrm{CaCu}_{2} \mathrm{O}_{8+\delta}$ single crystals," Physica C, vol. 366, pp. 254-262, Feb. 2002.

[8] L. Forro and J. R. Cooper, "Effect of vacuum annealing on the structure and superconductivity of $\mathrm{Bi}_{2} \mathrm{Sr}_{2} \mathrm{CaCu}_{2} \mathrm{O}_{8+\delta}$ single crystals," Physica C, vol. 366, pp. 254-262, Feb. 2002.

[9] Y. Ando, Y. Kurita, S. Komiya, S. Ono, and K. Segawa, "Evolution of the hall coefficient and the peculiar electronic structure of the cuprate superconductors," Phys. Rev. Lett., vol. 92, pp. 197001-1-197001-4, May 2004.
[10] K. Segawa and Y. Ando, "Intrinsic hall response of the $\mathrm{CuO}_{2}$ planes in a chain-plane composite system of $\mathrm{YBa}_{2} \mathrm{Cu}_{3} \mathrm{O}_{y}$," Phys. Rev. B, vol. 69, pp. 104521-1-104521-8, Mar. 2004.

[11] Z. Konstantinovic, Z. Z. Li, and H. Raffy, "Temperature dependence of the resistivity of oxygen controlled $\mathrm{Bi}_{2} \mathrm{Sr}_{2} \mathrm{CaCu}_{2} \mathrm{O}_{8+\delta}$ thin films: Pseudogap effect," Physica C, vol. 259-261, pp. 567-568, Mar. 1999.

[12] V. J. Emery and S. A. Kivelson, "Superconductivity in bad metals," Phys. Rev. Lett, vol. 74, pp. 3253-3332, Apr. 1995.

[13] K. Semba and A. Matsuda, "Superconductor-to-Insulator Transition and Transport Properties of Underdoped $\mathrm{YBa}_{2} \mathrm{Cu}_{3} \mathrm{O}_{y}$ Crystals," Phys. Rev. Lett, vol. 74, pp. 3253-3256, Apr. 1995.

[14] Z. Konstantinovic, Z. Z. Li, and H. Raffy, "Temperature dependence of the Hall effect in single-layer and bilyaer $\mathrm{Bi}_{2} \mathrm{Sr}_{2} \mathrm{Ca}_{n-1} \mathrm{Cu}_{n} \mathrm{O}_{y}$ thin films at various oxygen contents," Phys. Rev. B, vol. 62, pp. R11989-R11992, Nov. 2000.

[15] Y. Ando, A. N. Lavrov, S. Komiya, K. Segawa, and X. F. Sun, "Mobility of the doped holes and the antiferromagnetic correlations in underdoped high- $T_{c}$ cuprates," Phys. Rev. Lett, vol. 87, pp. 0170011-017001-4, Apr. 2001.

[16] Y. Ando, S. Komiya, K. Segawa, S. Ono, and Y. Kurita, "Electronic phase diagram of high- $T_{c}$ cuprate superconductors from a mapping of the in-plane resistivity curvature," Phys. Rev. Lett, vol. 93, pp. 2670011-267001-4, Dec. 2004.

[17] Y. He, T. S. Nunner, P. J. Hirschfeld, and H.-P. Cheng, "Local electronic structure of $\mathrm{Bi}_{2} \mathrm{Sr}_{2} \mathrm{CaCu}_{2} \mathrm{O}_{8}$ near oxygen dopants: A window on the high- $T_{c}$ pairing mechanism," Phys. Rev. Lett, vol. 96, pp. 197002-1-197002-4, May 2005.

[18] K. McElroy, J. Lee, J. A. Slezak, D.-H. Lee, H. Eisaki, S. Uchida, and J. C. Davis, "Atomic-scale sources and mechanism of nanoscale electronic disorder in $\mathrm{Bi}_{2} \mathrm{Sr}_{2} \mathrm{CaCu}_{2} \mathrm{O}_{8+\delta}$," Science, vol. 309, pp. 1048-1052, Aug. 2005. 